\title{
7. GENERATION OF ACOUSTIC NOISE IN CONVECTION ZONES
}

\author{
W. Unno*
}

\section{(University of Tokyo)}

In the Bakerian Lecture I96t, Lighthill (I) summarized the basic studies carried out by himself and his colleages on aerodynamical generation of sound in a homogeneous medium. In stellar convection zones, however, the characteristic size of a turbulent element is considered to be larger than the scale height so that the inhomogeneity due to gravity cannot be neglected in the problem. Recently, this problem has been studied very carefully by Moore and Spiegel (2). The detailed mechanism of noise generation, however, was rather assumed than deduced from the characteristics of turbulence. The latter problem was taken up in a confusing way by Unno and Kato (3) and Kato (4).

The basic equations of the problem are the equations of motion, continuity and energy (viscosity being neglected),

$$
\begin{gathered}
\rho_{0} \frac{\partial \mathbf{u}}{\partial \mathrm{t}}+\nabla \rho_{1}-\rho_{\mathbf{1}} \mathbf{g}=\mathbf{f}, \\
\frac{\partial \rho_{1}}{\partial \mathrm{t}}+\nabla \cdot\left(\rho_{0} \mathbf{u}\right)=q,
\end{gathered}
$$

and

$$
\frac{\partial \rho_{1}}{\partial t}+\rho_{0} \mathbf{g} \cdot \mathbf{u}+\gamma \rho_{0} \nabla \cdot \mathbf{u}=h
$$

where

and

$$
\begin{aligned}
& \mathbf{f}=-\frac{\partial\left(\rho \mathbf{u} u_{j}\right)}{\partial x_{j}}-\frac{\partial\left(\rho_{1} \mathbf{u}\right)}{\partial \mathrm{t}}, \\
& q=-\nabla \cdot\left(\rho_{1} \mathbf{u}\right),
\end{aligned}
$$

$$
h=-\gamma p_{1} \nabla \cdot \mathbf{u}-\mathbf{u} \cdot \nabla p_{1} .
$$

Symbols with suffix $\circ$ and $\mathbf{I}$ denote mean quantities and perturbations, respectively; $\mathbf{f}, q$ and $h$ represent non-linear terms in fluctuating quantities. Symbols which have usual meanings are not defined here. Radiative effects are not studied here, although they are important in the convection zone. For the general case with gravity, the solution of the inhomogeneous wave equation is hard to obtain. The circumstances are much simplified, if we assume isothermal disturbances $(\gamma=1)$ in an isothermal atmosphere. Although the treatment which follows can be generalized to the case of $\gamma \neq \mathrm{I}$, we shall restrict our study to the simplest case. Gravity waves cannot be studied then, but the characteristics of acoustic waves under the influence of gravity can be seen in the simplest form.

In order to compare closely with the analysis by Lighthill (5), we choose a modified density variation as the field variable:

$$
\rho^{\prime}(\mathbf{x}, t)=p_{0}^{-t}(z) \rho_{1}(\mathbf{x}, t)
$$

Eliminating the linear terms in $\mathbf{u}$ and $p$, among equations ( 1 ), (2), and (3), we obtain

$$
\left(\frac{\partial^{2}}{\partial t^{2}}-c^{2} \Delta^{2}+\frac{g^{2}}{4 c^{2}}\right) \rho^{\prime}(\mathbf{x}, t)=S(\mathbf{x}, t),\left(c^{2}=p_{0} / \rho_{0}\right)
$$

* This work was performed during the stay in the Max-Planck-Institut für Physik und Astrophysik, München (Germany). 
where

$$
S(\mathbf{x}, t)=-p_{0}^{-\frac{1}{2}}\left[\nabla \cdot \mathbf{f}+\left(\frac{\partial}{\partial t}\right)^{-1} \mathbf{g} \cdot \nabla q-\frac{\mathbf{I}}{c^{2}}\left(\frac{\partial}{\partial t}\right)^{-1}\left(\frac{\partial^{2}}{\partial t^{2}}+\mathbf{g} \cdot \nabla\right) h\right]
$$

Using equations (4), (5), and (6) neglecting terms of higher order than the second and omitting those non-linear terms which appear in the same form as the left-hand side (propagation part) of equation (8), we obtain

$$
\begin{aligned}
S(\mathbf{x}, t) & =p_{0}^{-\frac{1}{2}} \frac{\partial^{2}\left(\rho_{0} u_{i} u_{j}\right)}{\partial x_{i} \partial x_{j}} \\
& =\frac{1}{c}\left[\frac{\partial^{2}\left(\rho_{0}^{-\frac{1}{2}} u_{i} u_{j}\right)}{\partial x_{i} \partial x_{j}}-\frac{g}{c^{2}} \frac{\partial\left(\rho_{0}^{\frac{1}{2}} w u_{j}\right)}{\partial x_{j}}+\frac{g^{2}}{4 c^{2}}\left(\rho_{0}^{\frac{1}{2}} w w\right)\right]
\end{aligned}
$$

where

$$
\mathbf{g}=(\circ, \circ,-g) \text { and } \mathbf{u}=\left(u_{1}, u_{2}, w\right) \text {. }
$$

The source term $S(\mathbf{x}, t)$ consists of three terms which differ from each other in the degree of space differentiation. The first, the second, and the third terms express quadrupole, dipole and monopole sources, respectively. Attention should be paid for the fact that the inhomogeneity acts like the boundary surface of the homogeneous case (Curle (6)) and gives rise to dipole and monopole sources. The resolution of equation (8) is obtained for the Fourier amplitude of $\rho^{\prime}$ which is defined by

$$
\rho_{\sigma}(\mathbf{x})=\frac{\mathbf{I}}{2 \pi} \int_{-\infty}^{\infty} \mathrm{e}^{-i \sigma^{\prime} t} \rho^{\prime}\left(\mathbf{x}, t^{\prime}\right) d t^{\prime} \text { then } \rho^{\prime}(\mathbf{x}, t)=\int_{-\infty}^{\infty} \mathrm{e}^{i \sigma^{\prime} t} \rho_{\sigma^{\prime}}(\mathbf{x}) \mathrm{d} \sigma^{\prime}
$$

We obtain, after some partial integrations,

$$
\begin{aligned}
\rho_{0}(\mathbf{x}) & =\frac{\mathrm{I}}{8 \pi^{2} c^{2}} \int_{\mathrm{V}} \frac{\mathrm{d} \mathbf{x}^{\prime}}{R^{\prime}} \mathrm{e}^{-i \frac{\sigma_{1}}{\theta} R^{\prime}} \int_{-\infty}^{\infty} \mathrm{d} t^{\prime} \mathrm{e}^{-i \sigma t^{\prime}} \mathrm{S}\left(\mathbf{x}^{\prime}, t^{\prime}\right) \\
& =\frac{\mathrm{I}}{8 \pi^{2} c^{5}} \int_{\mathrm{V}} \frac{\mathrm{d} \mathbf{x}^{\prime}}{R^{\prime}} \int_{-\infty}^{\infty} \mathrm{d} t^{\prime} \mathrm{e}^{-i\left(\sigma t^{\prime}+\frac{\sigma_{1}}{c} R^{\prime}\right)} \rho_{0}\left(\mathbf{x}^{\prime}\right)^{\frac{1}{2}}\left[-\sigma_{1} n_{i}^{\prime} n_{j}^{\prime} u_{i} u_{j}^{\prime}+i 2 \sigma_{0} \sigma_{1} n_{j}^{\prime} w^{\prime} u_{j}^{\prime}+\sigma_{0}^{2} w^{\prime 2}\right]
\end{aligned}
$$

where

$$
\begin{gathered}
\sigma_{1}^{2}=\sigma^{2}-\sigma_{0}^{2}, \sigma_{0}^{2}=g^{2} /\left(4 c^{2}\right), \sigma_{1} \sigma>\circ(\text { radiation condition), } \\
\mathbf{R}^{\prime}=\mathbf{x}-\mathbf{x}^{\prime}, \quad R^{\prime}=\left|\mathbf{R}^{\prime}\right|
\end{gathered}
$$

and

$$
\mathbf{u}\left(\mathbf{x}^{\prime}, t^{\prime}\right)=\left(u_{1}^{\prime}, u_{2}^{\prime}, w^{\prime}\right), \quad \mathbf{n}^{\prime}=\mathbf{R}^{\prime} / R^{\prime}=\left(n_{1}^{\prime}, n_{2}^{\prime}, n_{3}^{\prime}\right),
$$

The acoustic intensity is derived from the energy conservation and is given by

$$
\left.I(\mathbf{x})=\left\langle p_{1} \mathbf{u}\right\rangle=-c^{6}<\rho^{\prime}\left(\frac{\partial}{\partial t}\right)^{-1}\left(\nabla-\frac{\mathbf{I}}{2 c^{2}} \mathbf{g}\right) \rho^{\prime}\right\rangle .
$$

At large distance from the source, we have

$$
\begin{aligned}
& I(\mathbf{x})=\frac{\mathbf{I}}{3^{2 \pi^{3}} \mathrm{c}^{4} R^{2}} \int_{\mathrm{V}} \mathrm{d} \mathbf{x}_{0} \rho_{0}\left(\mathbf{x}_{0}\right) \int_{|\sigma|>\sigma_{0}} \mathrm{~d} \sigma \int_{\mathrm{V}} \mathrm{d} \mathbf{r} \int_{-\infty}^{\infty} \mathrm{d} \tau \mathrm{e}^{i\left[\sigma \tau-\left(\sigma_{\mathbf{2}} / c\right) n . r\right]} \\
& +\frac{\mathbf{I}}{\sigma}\left(\frac{\sigma_{1}}{c} \mathbf{n}+i \frac{\mathrm{I}}{2 c^{2}} \mathbf{g}\right)\left(C_{\mathrm{n}}+\mathrm{i} S_{\mathbf{n}}\right)
\end{aligned}
$$


where

$$
\begin{gathered}
C_{\mathbf{n}}\left(\mathbf{x}_{0} ; \mathbf{r}, \tau\right)=\sigma_{1}^{4} n_{i} n_{j} n_{l} n_{m}<u_{i}^{\prime} u_{j}^{\prime} u_{l} u_{m}>\text { (quadrupole) } \\
+\sigma_{0}^{2} \sigma_{1}^{2}\left[4 n_{j} n_{m}<w^{\prime} u_{j} w^{\prime \prime} u_{m}>\right.\text { (dipole) } \\
\left.-\left(n_{i} n_{j}<u_{i}^{\prime} u_{j}^{\prime} w^{\prime 2}>+n_{l} n_{m}<w^{\prime 2} u_{l} u_{m}^{\prime \prime}>\right)\right] \text { (interference) } \\
+\sigma_{0}^{4}<w^{\prime 2} w^{\prime 2}>, \quad(\text { monopole) } \\
S_{\mathbf{n}}\left(\mathbf{x}_{0} ; \mathbf{r}, \tau\right)=-\sigma_{0} \sigma_{1}\left(\sigma_{1}^{2} n_{j} n_{m}+\sigma_{0}^{2} \delta_{j 3} \delta_{m 3}\right) \times \\
\times\left(<w^{\prime} u_{i}^{\prime}\left(\mathbf{n} \cdot \mathbf{u}^{\prime \prime}\right) u_{m}^{\prime \prime}>-<\left(\mathbf{n} \cdot \mathbf{u}^{\prime}\right) u_{j}^{\prime} w^{\prime \prime} u_{m}^{\prime \prime}>\right), \quad \text { (interference) } \\
\mathbf{x}_{0}=\left(\mathbf{x}^{\prime}+\mathbf{x}^{\prime \prime}\right) / \mathbf{2}, \mathbf{r}=\mathbf{x}^{\prime \prime}-\mathbf{x}^{\prime}, \tau=t^{\prime \prime}-t^{\prime}, \mathbf{R}=\mathbf{n} R=\mathbf{x}-\mathbf{x}_{0},
\end{gathered}
$$

and the fourth order velocity correlations $\left\langle u_{i}^{\prime} u_{j}^{\prime} u_{l}^{\prime \prime} u_{m}^{\prime \prime}\right\rangle$ are taken between two points $\left(x^{\prime}, t^{\prime}\right)$ and $\left(x^{\prime \prime}, t^{\prime \prime}\right)$. The terms proportional to $\mathbf{g}$ in equation (18) vanish because $C_{\mathrm{n}}$ and $S_{\mathrm{n}}$ are symmetric and antisymmetric for the exchange of two points, and are even and odd functions of $\sigma$, respectively. Neglecting $S_{\mathrm{n}}$ which is small compared with $C_{\mathrm{n}}$, and neglecting also $\left(\sigma_{1} / c\right) \mathbf{n} \cdot \mathbf{r}$ in the exponent in equation (18) which is small at low Mach number compared with $\sigma \tau$, we obtain finally

$$
\begin{array}{r}
I(\mathbf{x})=\frac{\mathbf{n}}{\mathrm{I} 6 \pi^{3} R^{2}} \int_{\mathrm{V}} \mathrm{d} \mathbf{x}_{0} \rho_{0}\left(\mathbf{x}_{0}\right) \int_{0}^{\infty} \mathrm{d} \sigma_{1} \int \mathrm{d} \mathbf{r} \int_{-\infty}^{\infty} \mathrm{d} \tau \cos (\sigma \tau) \frac{\sigma_{1}^{2} / \mathbf{c}^{5}}{\sigma_{1}^{2}+\sigma_{0}^{2}}\left(f_{q} \sigma_{1}^{4}+f_{d} \sigma_{0}^{2} \sigma_{1}^{2}+\sigma_{0}^{4}\right) \\
<w^{\prime 2} w^{\prime 2}>
\end{array}
$$

where the factors $f_{q}(\mathbf{n})$ and $f_{d}(\mathbf{n})$ account for multiplicities of respective sources.

To illustrate the nature of the various terms contributing to $I(\mathbf{x})$, let us adopt an idealized form of the correlation function, namely

$$
<w^{2} w^{\prime \prime 2}>=u_{0}^{4} \exp \left[-(\mathrm{r} / 2)\left(r^{2}+u_{0}^{2} \tau^{2}\right) / l_{0}^{2}\right]
$$

where $u_{0}$ and $l_{0}$ denote the characteristic velocity and length of turbulence.

Then we obtain

$$
I(\mathbf{x})=\frac{\sqrt{2 \mathbf{n}}}{4 \pi R^{2}} \int_{\mathrm{V}} \mathrm{d} \mathbf{x}_{0} \frac{\rho_{0} u_{0}^{3} l_{0}^{4}}{c^{5}} \int_{0}^{\infty} \mathrm{e}^{-\frac{1}{2}\left(\frac{l_{0}}{u_{0}}\right)^{2}\left(\sigma_{\perp}^{2} \sigma_{0}^{2}\right)} \frac{\sigma_{1}^{2}}{\sigma_{1}^{2}+\sigma_{0}^{2}}\left(f_{q} \sigma_{1}^{4}+f_{d} \sigma_{0}^{2} \sigma_{1}^{2}+\sigma_{0}^{4}\right) \mathrm{d} \sigma_{1}
$$

For laboratory cases in which $l_{0}<2\left(u_{0} / c\right) H$, ( $H$ being the scale height), we have

$$
I(\mathbf{x}) \sim \frac{\mathbf{n}}{4 \pi R^{2}} \int_{\mathrm{v}} \mathrm{d} \mathbf{x}_{0} \frac{\sqrt{\pi}}{2} \frac{\rho_{0} u_{0}^{3}}{l_{0}}\left[6 f_{q}\left(\frac{u_{0}}{c}\right)^{5}+\frac{1}{2} f_{d}\left(\frac{u_{0}}{c}\right)^{3}\left(\frac{l_{0}}{H}\right)^{2}+\frac{1}{8}\left(\frac{u_{0}}{c}\right)\left(\frac{l_{0}}{H}\right)^{4}\right],
$$

where $f_{q}$ should be about 5 according to Proudman (7). Dipole and monopole radiations are negligible at low Mach number.

For stellar cases in which $l_{0}>2\left(u_{0} / c\right) H$, we have

$$
I(\mathbf{x}) \sim \frac{\mathbf{n}}{4 \pi R^{2}} \int_{\mathrm{V}} \mathrm{dx}_{0} \frac{\sqrt{\pi}}{2} \frac{\rho_{0} u_{0}^{3}}{l_{0}}\left[\operatorname{I} 20 f_{q}\left(\frac{u_{0}}{c}\right)^{7}\left(\frac{l_{0}}{H}\right)^{-2}+6 f_{d}\left(\frac{u_{0}}{c}\right)^{5}+\frac{1}{2}\left(\frac{u_{0}}{c}\right)^{3}\left(\frac{l_{0}}{H}\right)^{2}\right] \mathrm{e}^{-\frac{1}{8}\left(\frac{c l_{0}}{u_{0} H}\right)^{2}}
$$

The dipole and monopole are important at low Mach number. The acoustic power is greatly reduced by the last exponential factor. The conclusions drawn here depend very much on the assumption (23). Especially, the dependence on $\tau$ is crucial. To obtain a more precise representation, we shall have to await a suitable treatment of turbulent convection. 


\title{
REFERENCES
}

I. Lighthill, M. J. Proc. R. Soc., A 267, 147, 1962.

2. Moore, D. W., Spiegel, E. A. Astrophys. F., 139, 48, 1964.

3. Unno, W., Kato, S. Publ. astr. Soc. Yapan, 14, 416, 1962.

4. Kato, S. Publ. astr. Soc. Japan, 15, 204, 1963.

5. Lighthill, M. J. Proc. R. Soc., A 211, 564, x952.

6. Curle, N. Proc. R. Soc., A 23I, 505, 1955.

7. Proudman, I. Proc. $R$. Soc., A 214, I19, 1952.

\section{DISCUSSION}

$M$. Kuperus. Does the interference between the various multipole radiation fields occur in a constructive or a destructive way?

$W$. Unno. The interference between quadrupole and monopole radiations is mainly destructive. The interference of dipole radiation with quadrupole radiation and that with monopole radiations seems to cancel each other mostly.

P. A. Sturrock. Dr Unno's analysis is valid for that part of the spectrum with $\omega>\omega_{0}$, where $\omega_{0}=(c / 2 H)$. This part couples into travelling waves. However, that part of the turbulence spectrum with $\omega<\omega_{0}$ still couples into evanescent acoustic waves. Although the $\omega>\omega_{0}$ part is important for the heating of the upper chromosphere and corona, the $\omega<\omega_{0}$ part is probably important for the heating of the low corona. This part of problem therefore merits analysis.

W. Unno. Yes, I agree.

\section{TRAPPED WAVES IN THE SOLAR ATMOSPHERE}

\author{
F. D. Kahn
}

(University of Manchester)

The temperature of the solar atmosphere passes through a minimum at a certain layer in the photosphere. The speed of sound increases with distance from this layer, both above and below. Rays of sound tend, therefore, to be refracted back into the layer. For rays travelling at not too large an angle with the horizontal this leads to a continual refocusing of disturbances, at about 5-minute intervals. The approximations made are such as to render this description valid only for waves of small wavelength.

\section{REFERENCES}

Kahn, F. D. Astrophys. F., 134, 343, 196r; 135, 547, 1962.

\section{DISCUSSION}

$M . P$. Souffrin. How is the picture modified in the three-dimensional case, when refocusing occurs not at a point, but along an annulus? Is the characteristic size of the picture still given by the wavelength introduced by Dr Kahn?

F. Kahn. Linear disturbances can always be analysed into plane waves. Having dealt with the behaviour of plane waves by ray theory we can now build up solutions by superposition. 